\title{
Electrically small split ring resonator antennas
}

Kamil Boratay Alici, and Ekmel Ozbay

Citation: Journal of Applied Physics 101, 083104 (2007); doi: 10.1063/1.2722232

View online: http://dx.doi.org/10.1063/1.2722232

View Table of Contents: http://aip.scitation.org/toc/jap/101/8

Published by the American Institute of Physics

\section{Articles you may be interested in}

Split-ring resonator for use in magnetic resonance from $200-2000 \mathrm{MHz}$

Review of Scientific Instruments 52, 213 (1998); 10.1063/1.1136574

Split ring resonator-based left-handed coplanar waveguide

Applied Physics Letters 83, 4652 (2003); 10.1063/1.1631392

Split ring resonator sensors for infrared detection of single molecular monolayers

Applied Physics Letters 95, 043113 (2009); 10.1063/1.3194154

Electromagnetic resonances in individual and coupled split-ring resonators

Journal of Applied Physics 92, 2929 (2002); 10.1063/1.1497452

Tuned permeability in terahertz split-ring resonators for devices and sensors

Applied Physics Letters 91, 062511 (2007); 10.1063/1.2768300

An antenna-coupled split-ring resonator for biosensing

Journal of Applied Physics 116, 124701 (2014); 10.1063/1.4896261

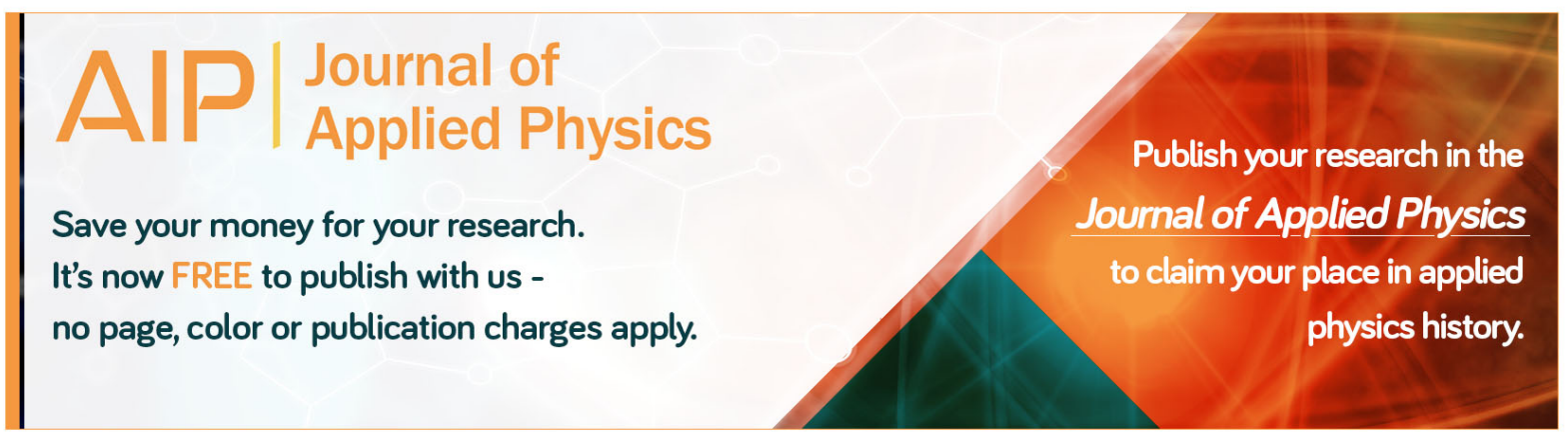




\title{
Electrically small split ring resonator antennas
}

\author{
Kamil Boratay Alicia and Ekmel Ozbay \\ Nanotechnology Research Center, Department of Physics, \\ Department of Electrical and Electronics Engineering, Bilkent University, 06800 Ankara, Turkey
}

(Received 21 January 2007; accepted 26 February 2007; published online 20 April 2007)

\begin{abstract}
We studied electrically small resonant antennas composed of split ring resonators (SRR) and monopoles. The antennas considered have the same ring radius, but slightly different geometry. The resonance frequency depends on the geometry of the SRRs. Two SRR antennas are designed. The first one, which operates at $3.62 \mathrm{GHz}$, is demonstrated theoretically and experimentally. The size of this antenna is $0.095 \lambda_{0} \times 0.100 \lambda_{0}$ and is low profile at the other dimension. The gain and directivity of the antenna was 2.38 and 5.46, respectively. The corresponding efficiency was $43.6 \%$. The estimated radiation $Q(\operatorname{rad} Q=23.03)$ was much larger than the minimum radiation $Q$ (min $Q=1.78)$. The second one is a rather small SRR antenna in which the capacitance between the rings is increased. The size is reduced to $0.074 \lambda_{0} \times 0.079 \lambda_{0}$. This structure is called serrated SRR (SSRR). Both antennas have similar far-field patterns but the efficiency of the SSRR antenna is less. (C) 2007 American Institute of Physics. [DOI: 10.1063/1.2722232]
\end{abstract}

\section{INTRODUCTION}

Electrically small antennas (ESAs) occupy a volume of the sphere whose radius is a small fraction of the free-space wavelength of the radiated electromagnetic field. ${ }^{1}$ The fundamental limitations of ESAs were investigated by Wheeler in $1947 .^{2}$ One year later, Chu derived a theoretical relationship between the dimensions of an antenna and its minimum

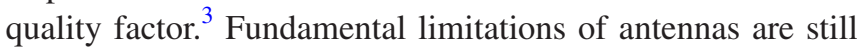
an active research area. ${ }^{4-6}$ The general techniques applied in order to achieve these theoretical limits are the optimization of the antenna topology, 7,8 and usage of magneto-dielectric materials. ${ }^{9,10}$

Artificial materials that exhibit unusual physical properties attracted much attention in the last decade of the last century. ${ }^{11}$ Can the introduction of these materials into antennas improve the current performance, especially in terms of their rather small size? The invention of antennas, which resonantly couple to external radiation, composed of single negative materials is a rather interesting study. ${ }^{12}$ Moreover, research on obtaining an electrically small size by using metamaterials has been presented in the literature recently. ${ }^{13-17}$ Eleftheriades et al. proposed a metamaterial ring antenna of efficiency 54\% (Ref. 16) and Stuart et al. theoretically demonstrated an electrically small antenna with $k a<0.5$. $^{17}$

One of the most important elements of metamaterials is the split ring resonator (SRR), which was designed by Pendry et al. in $1999 .^{18}$ The geometrical parameters of this structure determine its resonance frequency. The SRR can show resonant behavior at wavelengths that are much larger than its own size. Experimental demonstrations of these structures have been achieved by many researchers. ${ }^{19-21}$ In the present work, we explain a technique that uses the SRRs in order to obtain ESAs.

${ }^{a)}$ Electronic mail: bora@fen.bilkent.edu.tr

\section{EXPERIMENT}

The SRR is electrically excited with a monopole antenna. The configuration is shown in Fig. 1, in which the geometrical parameters of the structure are as follows: outer ring radius $r_{1}=3.6 \mathrm{~mm}$, inner ring radius $r_{2}=5 \mathrm{~mm}$, width of the strips $w=0.9 \mathrm{~mm}$, separation between the two adjacent rings $s=0.2 \mathrm{~mm}$, the length of the splits $g=0.2 \mathrm{~mm}$. Distance to the ground plane $h=0.8 \mathrm{~mm}$, the distance of the end of the radiating inner wire part to the top of the printed circuit board $(\mathrm{PCB}) t_{1}=0.2 \mathrm{~mm}$, the distance between the PCB and radiating inner wire part of the monopole $t_{2}=0.1 \mathrm{~mm}$, thickness of the ground plane $d_{1}=0.5 \mathrm{~mm}$, length of the coaxial cable above the ground plane (this part was necessary for physically strengthening the monopole) $d_{2}=0.5 \mathrm{~mm}$, PCB side length $h_{1}=7.8 \mathrm{~mm}$, and inner wire height $h_{2}=8.32 \mathrm{~mm}$. The coaxial cable has four major parts: the inner wire with a radius of $0.245 \mathrm{~mm}$, Teflon part with a thickness of $1.08 \mathrm{~mm}$, and a dielectric constant 2, conducting shield part with a thickness of $0.48 \mathrm{~mm}$ and insulator coating with $0.48 \mathrm{~mm}$ thickness.

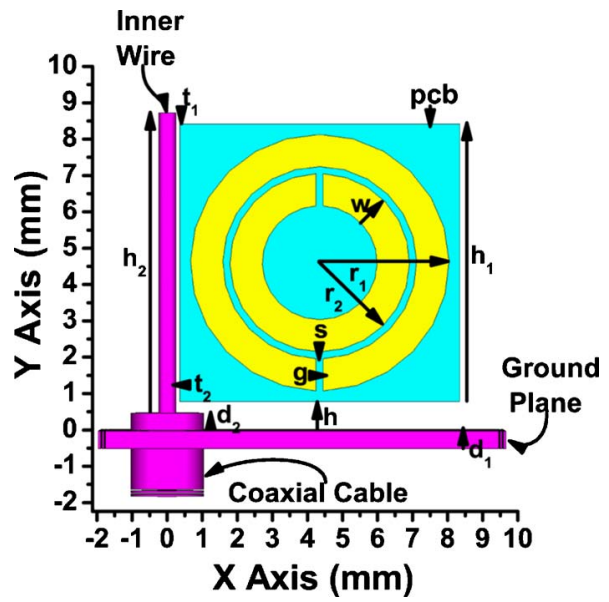

FIG. 1. The geometry of the SRR antenna is shown, but only a part of the ground plane and the coaxial cable. 


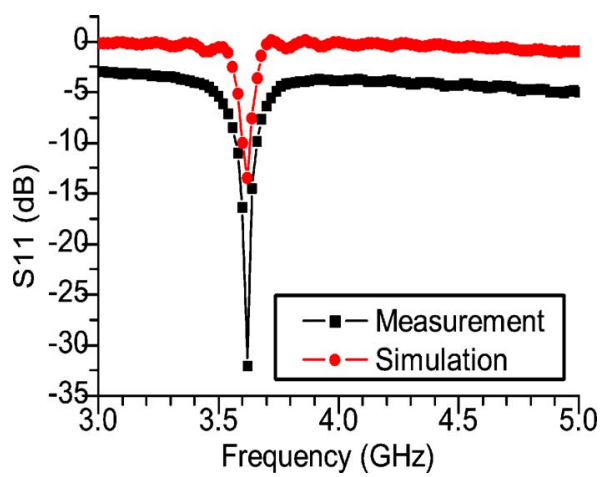

FIG. 2. $S_{11}$ of the SRR antenna, experiment, and simulation

The substrate PCB was standard FR-4 material with relative permittivity $\varepsilon_{r}=3.5$. The thickness of the PCB was 1.6 $\mathrm{mm}$ and the deposited copper thickness on the PCB was $30 \mu \mathrm{m}$. SRR was obtained by properly etching the metal deposit of the PCB. The ground plane is nearly squareshaped at the $x-z$ plane, with the square side length being equal to the free-space wavelength $\left(\lambda_{0}\right)$ at the operation frequency $\left(f_{0}\right)$. The operation frequency is selected to be the frequency at which the SRR shows resonant behavior. This frequency is determined as $3.62 \mathrm{GHz}$ by considering the minimum of the input reflection coefficient, $S_{11}$. The antenna is simulated by using the commercial software Computer Simulation Technology, Microwave Studio (CST Mws), which is based on the finite integration method. The experiments are performed via an HP8510C network analyzer. The simulation and experiment data of the $S_{11}$ are presented in Fig. 2. The minimum of the $S_{11}$ data of the reflection experiment was $-32 \mathrm{~dB}$.

\section{RESULTS AND DISCUSSION}

The overall size of the antenna part is $0.095 \lambda_{0}$ $\times 0.100 \lambda_{0}$. Since the antenna part is above a conducting plane while defining the radius of the minimum sphere that encloses the antenna, we should also, therefore, consider the antenna's image. ${ }^{6}$ Therefore, the minimum radius $a$ for our antenna is $0.144 \lambda_{0}$, i.e., $11.95 \mathrm{~mm}$.
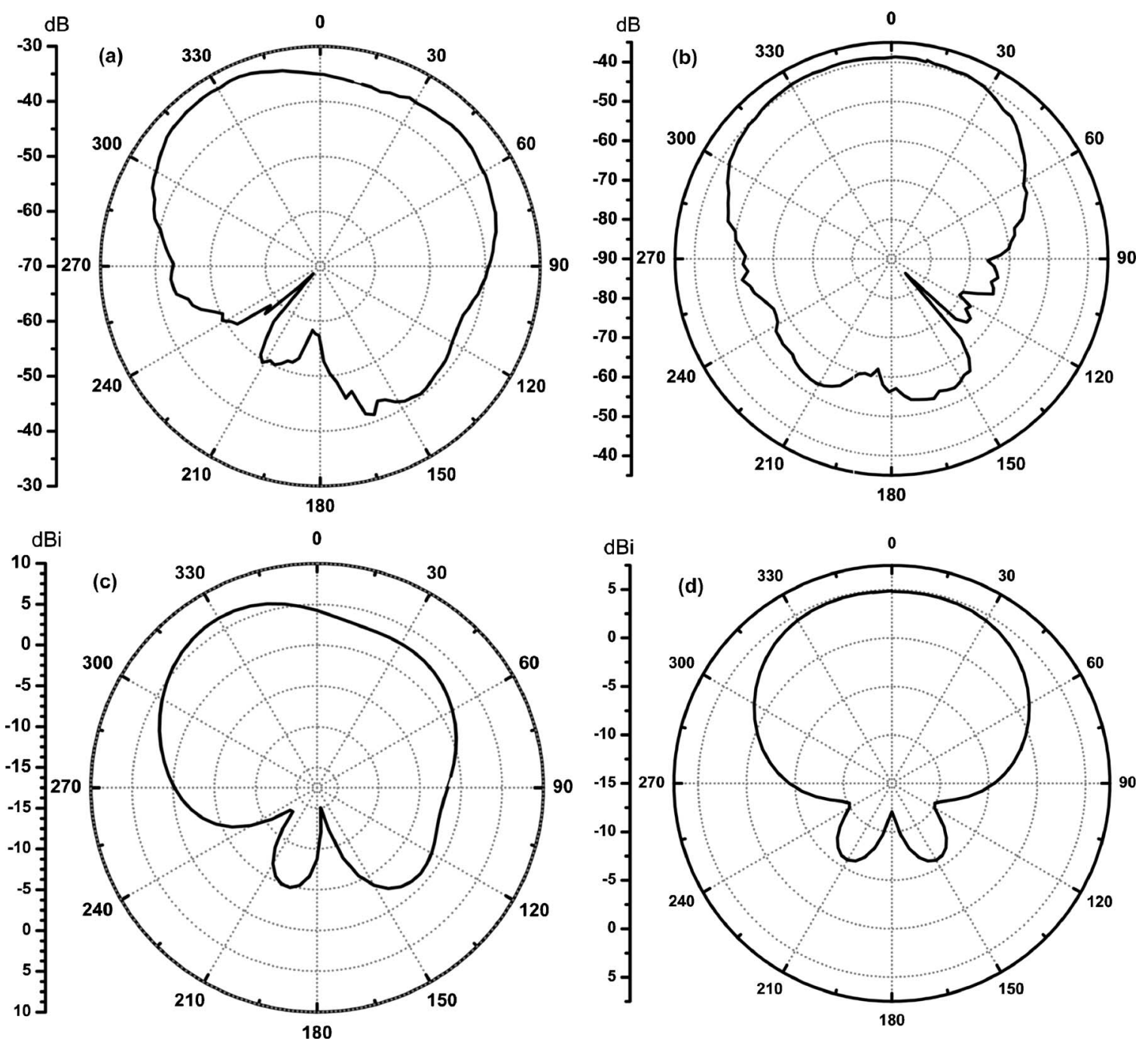

FIG. 3. Far-field radiation patterns of the SRR antenna. (a) E-plane measured ( $x-y$ plane); (b) $H$-plane measured ( $y$ - $z$ plane); (c) $E$-plane simulated; (d) $H$ - plane simulated. 
TABLE I. The figures of merit of some of the antennas.

\begin{tabular}{|c|c|c|c|c|c|c|c|c|c|c|c|}
\hline & $\begin{array}{l}\text { Freq. } \\
(\mathrm{GHz})\end{array}$ & Size & FBW & $\operatorname{Rad} Q$ & $\operatorname{Min} Q$ & $\begin{array}{c}-10 \mathrm{~dB} \\
\mathrm{BW}\end{array}$ & $a$ & $G_{\max }$ & Gain & $D_{0}$ & Efficiency \\
\hline SRRA Expt. & 3.62 & $0.095 \lambda_{0} \times 0.100 \lambda_{0}$ & 0.043 & 23.03 & 1.774 & $2.42 \%$ & $0.144 \lambda_{0}$ & 4.51 & 2.38 & 5.90 & $43.6 \%$ \\
\hline SRRA Sim. & 3.62 & $0.095 \lambda_{0} \times 0.100 \lambda_{0}$ & 0.028 & 36.20 & 1.774 & $0.88 \%$ & $0.144 \lambda_{0}$ & 4.51 & 3.11 & 4.95 & $62.8 \%$ \\
\hline SSRRA Sim. & 2.84 & $0.074 \lambda_{0} \times 0.079 \lambda_{0}$ & 0.029 & 34.63 & 2.795 & $0.35 \%$ & $0.113 \lambda_{0}$ & 2.33 & 1.06 & 5.63 & $18.8 \%$ \\
\hline
\end{tabular}

The radiation quality factor of an ESA is of fundamental interest. It is defined as $2 \pi$ times the ratio of the maximum energy stored to the total energy lost per period. ${ }^{3,4}$ We estimate the minimum radiation $Q$ of our antenna by using the following equation: ${ }^{4}$

$$
Q=\frac{1}{2}\left(\frac{1}{k^{3} a^{3}}+\frac{2}{k a}\right) \text {, }
$$

where $k$ is the wave number associated with the electromagnetic field operating at $f_{0}$ frequency. Therefore, we estimate the minimum radiation quality factor of the antenna as $\min Q=1.78$.

The $Q$ of the antenna was calculated by using the Foster's reactance theorem. ${ }^{5}$ The fractional bandwidth (FBW) and $-10 \mathrm{~dB}$ BW are obtained from the experimental $S_{11}$ data as 0.043 and $2.42 \%$, respectively. Therefore, the $Q \sim 1 / B W$ is 23.03 and is adequately large $\mathrm{e}^{22}$ and well above the theoretical limit.

The far-field radiation pattern measurements were performed by using a standard gain horn antenna as a receiver. The distance between the antennas was $\sim 1 \mathrm{~m}$, which corresponds to the far-field region for our antenna. The measured and simulated copolarized $E$ - and $H$-plane far-field radiation patterns are presented in Fig. 3. We see that the simulated and measured far-field patterns are similar; however, the simulation predicts symmetric sidelobe levels for the $H$-plane pattern. We were unable to see this symmetry due to experimental limitations. The value of the maximum directivity is approximately equal to ${ }^{23}$

$$
D_{0}=\frac{41253}{\Theta_{1 d} \times \Theta_{2 d}},
$$

where $\Theta_{1 d}$ is the half-power beamwidth in one plane and $\Theta_{2 d}$ in the perpendicular plane to the first, in degrees. The mea- sured half power beamwidth was $76^{\circ}$ along the $E$-plane and was $92^{\circ}$ along the $H$-plane. The absolute gain measurements are followed by the two antenna method calculations, in which we obtained the antenna gain as $G=2.38$. The corresponding radiation efficiency of the antenna was $43.6 \%$. The figures of merit of the antenna obtained from the measurements and simulation are summarized in Table I.

Finally, we should compare the gain of our antenna with the fundamental limit given by Fante. ${ }^{24}$ The maximum possible gain for our antenna estimated from the Fante's analysis is 4.51 . We see that our gain is less than the theoretical limit.

If we can excite the SRR electrically at rather small frequencies, the antenna size can be further miniaturized since the SRR resonance frequency can be tuned by increasing the capacitance between the rings. Considering this fact we designed serrated SRR, which has a resonance at $2.84 \mathrm{GHz}$. Just by changing the shape of the rings as shown in Fig. 4 we obtained a smaller SRR antenna. However, though the size is smaller, the maximum theoretical gain is thereby reduced to 2.33. Correspondingly, the antenna efficiency was then $18.8 \%$. The calculated figures of merit are presented in Table I. The simulation results for the insertion loss and the farfield radiation patterns are shown in Figs. 4 and 5, respectively.

\section{CONCLUSION}

We conclude that metamaterials can play a role in the development of ESAs. By electrically exciting the SRRs placed on a ground plane we were able to obtain resonant antennas with efficiencies exceeding $40 \%$. The sizes of the antenna were less than $\lambda_{0} / 10$.

We observed that as we continued decreasing the resonant frequency of the SRRs, the maximum theoretical gain and simultaneously the gain decreased. Therefore, we can estimate the limit of our method, which is used to miniatur-
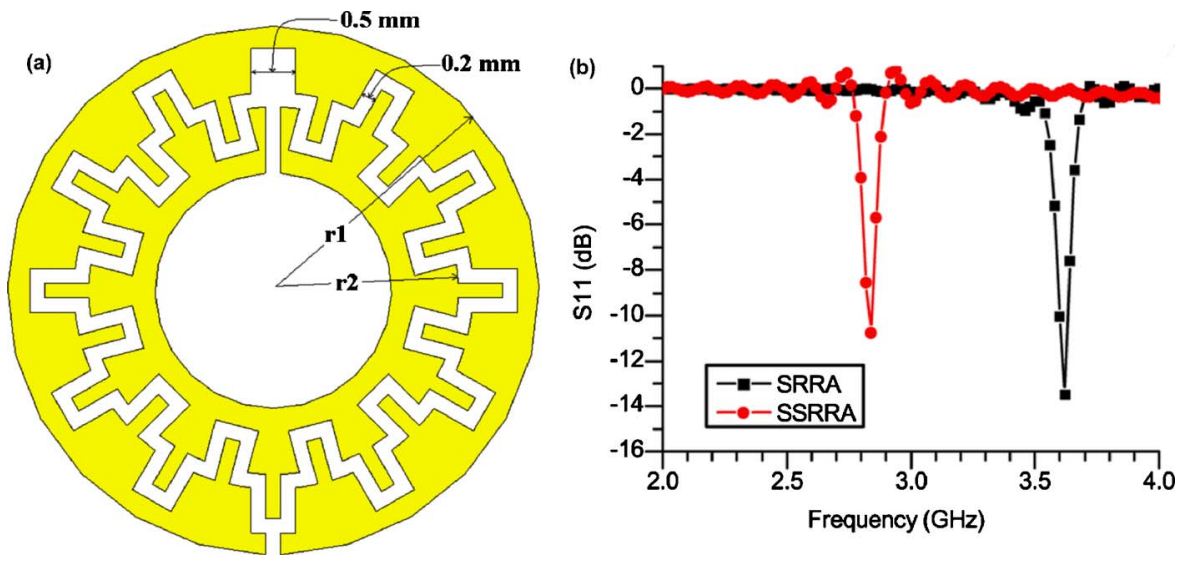

FIG. 4. (a) Serrated SRR geometry; (b) Return loss for the SSRR antenna and SRR antennas. 

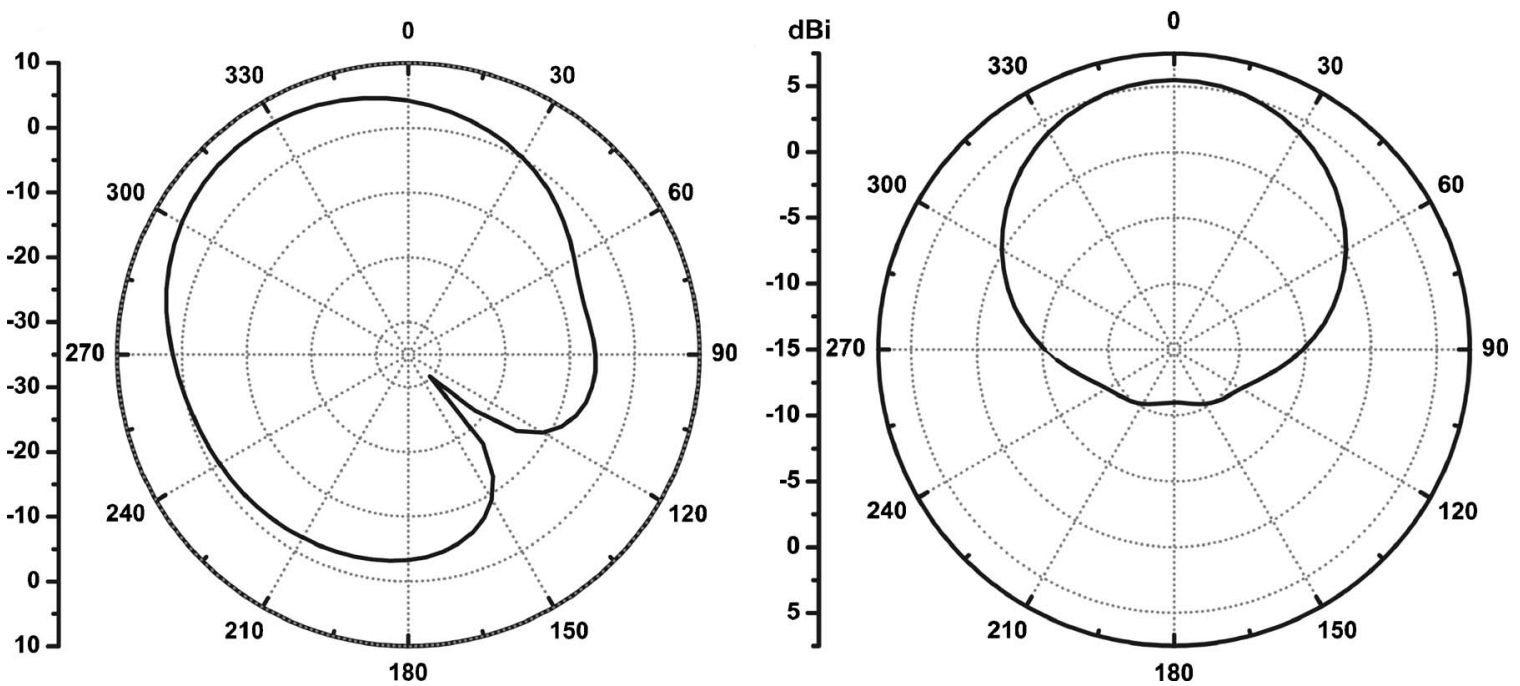

FIG. 5. (a) $E$ - plane and (b) $H$-plane simulated patterns of the SSRR antenna.

ize antennas. Moreover, it is important to note that when excited properly, SRRs above a ground plane radiate efficiently. These results can have applications in future wireless systems and in the development of the steerable phased array antennas.

\section{ACKNOWLEDGMENTS}

This work is supported by the European Union under the projects EU-DALHM, EU-NOE-METAMORPHOSE, EU-NOE-PHOREMOST, and TUBITAK under Project Nos. 104E090, 105E066, and 105A005. One of the authors (Ekmel Ozbay) also acknowledges partial support from the Turkish Academy of Sciences.

${ }^{1}$ R. C. Hansen, Proc. IEEE 69, 170 (1981).

${ }^{2}$ H. A. Wheeler, Proc. IRE 49, 1479 (1947).

${ }^{3}$ L. J. Chu, J. Appl. Phys. 19, 1163 (1948).

${ }^{4}$ J. S. Mclean, IEEE Trans. Antennas Propag. 44, 672 (1996).

${ }^{5}$ W. Geyi, P. Jarmuszewski, and Y. Qi, IEEE Trans. Antennas Propag. 48, 401 (2000).

${ }^{6}$ J. C. E. Sten, A. Hujanen, and P. K. Koivisto, IEEE Trans. Antennas Propag. 49, 829 (2001).

${ }^{7}$ S. R. Best, IEEE Trans. Antennas Propag. 53, 502 (2005).

${ }^{8}$ E. E. Altshuler, IEEE Trans. Antennas Propag. 50, 297 (2002).

${ }^{9} \mathrm{~K}$. L. Wong, Compact and Broadband Microstrip Antennas (Wiley, New York, 2002).

${ }^{10}$ K. Buell, H. Mosallaei, and K. Sarabandi, IEEE Trans. Microwave Theory
Tech. 54, 135 (2006).

${ }^{11}$ J. Pendry, Nat. Mater. 5, 599 (2006).

${ }^{12}$ E. D. Isaacs, P. M. Platzman, and J. T. Shen, U.S. Patent No. 6,661,392 (9 December 2003).

${ }^{13}$ S. Hrabar, J. Bartolic, and Z. Sipus, IEEE Trans. Antennas Propag. 53, 110 (2005).

${ }^{14}$ M. E. Ermutlu, C. R. Simovski, M. K. Karkkainen, P. Ikonen, S. A. Tretyakov, A. A. Sochava, in International Workshop Antenna Technology: Small Antennas and Novel Metamaterials (IEEE, New York, 2005), p. 87.

${ }^{15}$ P. Ikonen, M. Karkkainen, and S. Tretyakov, Experimental study of a $\lambda / 2$-patch antenna loaded with an array of metasolenoids as artifical magnetic substrate (IEEE, New York, 2005).

${ }^{16}$ F. Qureshi, M. A. Antoniades, and G. V. Eleftheriades, IEEE Antennas Wireless Propag. Lett. 4, 333 (2005).

${ }^{17}$ H. R. Stuart and A. Pidwerbetsky, IEEE Trans. Antennas Propag. 54, 1644 (2006).

${ }^{18}$ J. B. Pendry, A. J. Holden, D. J. Robbins, and W. J. Stewart, IEEE Trans. Microw. Theory Tech. 47, 2075 (1999).

${ }^{19}$ D. R. Smith, W. J. Padilla, D. C. Vier, S. C. Nemat-Nasser, and S. Schultz, Phys. Rev. Lett. 84, 4184 (2000).

${ }^{20}$ A. A. Houck, J. B. Brock, and I. L. Chuang, Phys. Rev. Lett. 90, 137401 (2003).

${ }^{21}$ O. Moser, B. D. F. Casse, O. Wilhelmi, and B. T. Saw, Phys. Rev. Lett. 94, 063901 (2005).

${ }^{22}$ G. A. Thiele, P. L. Detweiler, and R. P. Penno, IEEE Trans. Antennas Propag. 51, 1263 (2003).

${ }^{23}$ C. A. Balanis, Antenna Theory: Analysis and Design (Wiley, New York, 1997).

${ }^{24}$ R. Fante, IEEE Trans. Antennas Propag. 40, 1586 (1992). 